\title{
Special Issue on Current Topics in Calorimetry and Thermal Analysis in Poland
}

Published online: 4 August 2016

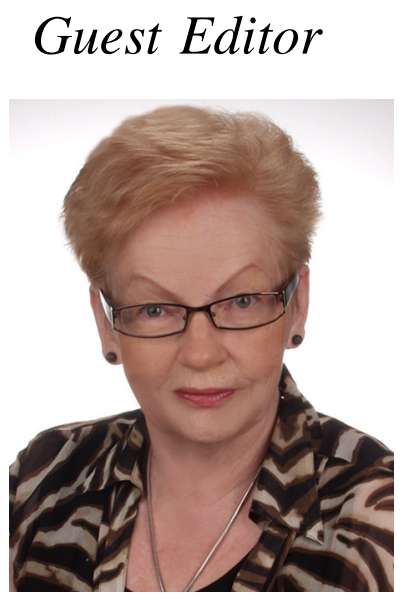

Prof. Barbara Pacewska

Faculty of Civil Engineering,

Mechanics and Petrochemistry,

Institute of Chemistry,

Warsaw University of Technology,

Płock, Poland

bpacewska@pw.plock.pl 


\title{
Preface
}

\author{
Barbara Pacewska ${ }^{1}$
}

(C) The Author(s) 2016. This article is published with open access at Springerlink.com

Conferences on Calorimetry and Thermal Analysis (CCTA) are the main scientific events co-organized by Polish Society of Calorimetry and Thermal Analysis (PTKAT) every 3 years in Zakopane (Poland), a beautiful town in the Tatra Mountains. These scientific meetings give possibility to present current trends in using thermal methods mainly in Poland, scientific discussions and also initiation of new cooperations. The conferences are of great interest of researchers from Poland and also from abroad.

This Special Issue of Journal of Thermal Analysis and Calorimetry (two parts: vol 125 no 3 and vol 126 no 1 , 2016) contains selected papers the results of which were previously presented in Zakopane (Poland) during CCTA 12-12th Conference on Calorimetry and Thermal Analysis organized together with 5th Joint Czech-Hungarian-Polish-Slovakian Thermoanalytical Conference (September 6-10, 2015). Faculty of Chemistry Maria CurieSkłodowska University (Lublin, Poland) was the main organizer of the conferences. Prof. Zofia Rzączyńska from the university mentioned above undertook the function of Conference Chairperson, while Prof. Barbara Pacewska (Warsaw University of Technology, Poland) was the Conference Co-Chairperson.

The main aim of the conferences was to give possibility of discussions on the results obtained by calorimetry and thermal analysis methods in different fields of investigations, exchange of scientific ideas and establishing new cooperation between researchers from Poland but also from other countries. The scientific program included plenary and session lectures, as well as oral communications and posters (in total ca. 320 contributions) presented in ten

Barbara Pacewska

bpacewska@pw.plock.pl

1 Faculty of Civil Engineering, Mechanics and Petrochemistry, Institute of Chemistry, Warsaw University of Technology, Plock, Poland scientific sections. The events also gave the opportunity for getting to know the technical innovations during exhibitions and lectures of instrument manufacturers.

The conferences were attended by 250 participants from different scientific institutes, including scientists from abroad, not only from Czech Republic, Slovak Republic and Hungary, but also from other 15 European (but not only) countries.

Opening ceremony of CCTA 12 was the opportunity for present the awards: Honorary Membership of PTKAT to Professors: Andrzej Małecki and Henryk Piekarski; Medal Memorial of W. Świętosławski to Professors: Judit Simon, Jerzy Błażejowski and Janusz Pysiak; PTKAT award for young scientists for their achievements in the field on calorimetry and thermal analysis to dr Mateusz Piz.

This Special Issue of Journal of Thermal Analysis and Calorimetry contains selected papers dedicated to the conferences. Each of the papers was evaluated by at least two independent reviewers. The papers cover a wide range of fields of applications of different thermoanalytical techniques used as main or complementary methods of analysis. I would like to express our gratitude to all the authors for submitting the manuscripts to this Special Issue.

Success of the conferences and this Special Issue is the result of work of many people. I appreciate time and great work of the reviewers. I am grateful to the members of the Organizing and Scientific Committees, especially to Prof. Zofia Rzączyńska, for their great effort during organization of the conferences. Many thanks for the great support of sponsors. My special thanks to Alfred Kállay-Menyhárd, Editor-in-Chief of JTAC, colleagues from the Editorial Office and Springer. Many thanks to all conference participants who provided high level of the contributions as well as friendly and unique atmosphere of the meeting. 


\section{Acknowledgements}

The Guest Editor is very thankful to the following scientists whose official reviews have significantly contributed to the quality of the papers in this Special Issue.

Abo Zeid, E. F., Egypt

Alghool Alghool, S., Egypt

Amim Jr., J., Brazil

Amin, S. K., Egypt

Androsch, R., Germany

Andrzejewska, E., Poland

Anyszka, R., Poland

Arena, G., Italy

Avar, B., Turkey

Avramov, I., Bulgaria

Bachaga, T., Tunisia

Badea, M. E., Romania

Balköse, D., Turkey

Bannach, G., Brazil

Barros, N., Spain

Bellavia, G., France

Bennici, S., France

Bikiaris, D., Greece

Birch, E. J., New Zealand

Blanco, I., Italy

Błońska-Tabero, A., Poland

Boczkowska, A., Poland

Bolbukh, Y., Ukraine

Borysiak, S., Poland

Bosacka, M., Poland

Bouza, R., Spain

Budrugeac, P., Romania

Burlett, D., USA

Caires Jr., F., Brazil

Cardinale, A. M., Italy

Carp, O., Romania

Cavalheiro, E., Brazil

Charmas, B., Poland

Chetehouna, K., France

Chibowski, S., Poland

Chow, W. S., Malaysia

Chrissafis, K., Greece

Chruściel, J. J., Poland

Cibulkova, Z., Slovakia

Circu, V., Romania

Costa Garcia, M., Brazil

Crespi, M. S., Brazil

Czeppe, T., Poland

Czylkowska, A., Poland

Dash, S., India

Datta, J., Poland

De Klerk, W., The Netherlands

Deja, J., Poland
Demetzos, C., Greece

Dettlaff, K., Poland

di Foggia, M., Italy

Ding, J., China

Doca, N., Romania

Dorosz, D., Poland

Drebushchak, V. A., Russian Federation

Drzazga, Z., Poland

Dudrova, E., Slovakia

Dweck, J., Brazil

Fernandez-Garcia, M., Spain

Fierascu, R. C., Romania

Filipek, E., Poland

Fima, P., Poland

Fukao, K., Japan

Gac, W., Poland

Galina, H., Poland

Garcia-Sanchez, E., Mexico

Gavrichev, K. S., Russian Federation

Gendvilas, R., Lithuania

Ghyngazov, S., Russian Federation

Giergiczny, Z., Poland

Godinho Jr., M., Brazil

Gołofit, T., Poland

Goncharuk, O., Ukraine

Gong, P., China

Gonzalez, E. P., Brazil

Goworek, J., Poland

Graczyk, T., Poland

Graziano, G., Italy

Gun'ko, V., Ukraine

Gurnule, W. B., India

Haponiuk, J., Poland

Hatakeyama, T., Japan

Hosny, N., Egypt

Howell, B. A., USA

Hubicki, Z., Poland

Hurduc, N., Romania

Jabłoński, M., Poland

Janotka, I., Slovakia

Janovec, J., Slovakia

Jóźwiak, M., Poland

Judovits, L., USA

Juszczak, L., Poland

Kareiva, A., Lithuania

Karolewicz, B., Poland

Karpiński, Z., Poland

Kasyap, S., India 
Keutgen, A., Austria

Khan Durrani, S., Pakistan

Kiseleva, D. V., Russian Federation

Kobelnik, M., Brazil

Koga, N., Japan

Kover, M., Slovakia

Kozłowska, M., Poland

Krumova, S., Bulgaria

Kumar S., India

Kumar T. V., United States

Lacomte-Nana, G. L., France

Łaccz, A., Poland

Lalia-Kantouri, M., Greece

Lehto, V. P., Finland

Lendzion-Bieluń, Z., Poland

Lerchner, J., Germany

Leveneur, S., France

Li, H., China

Li, J., China

Liška, M., Slovakia

Lisnyak, V. V., Ukraine

Liu, X., China

Logvinenko, V. A., Russian Federation

Lörinczy, D., Hungary

Lu, Q., China

Lubczak, J., Poland

Malek, J., Czech Republic

Markovic, G. S., Serbia

Marongiu, B., Italy

Melnikov, P., Brazil

Menapace, C., Italy

Mendes, L., C., Brazil

Mészáros Szécsényi, K., Serbia

Michnik, A., Poland

Mishra, N., India

Montedo, O. R. K., Brazil

Moreira Lima, C., Brazil

Moreno-Piraján, J. C., Colombia

Mosner, P., Czech Republic

Mousavian, R. T., Iran

Mustata, F., Romania

Nair, R., India

Njiomou Djangang, C., Cameroon

Nocun-Wczelik, W., Poland

Obranović, M., Croatia

Ostrowska-Ligeza, E., Poland

Ozao, R., Japan

Palou, M., Slovakia

Papageorgiou, G. Z., Greece

Pasierb, P., Poland

Pawlik, P., Poland

Pelovsky, Y., Bulgaria

Peppley, B., Canada
Pereira, R. N., Brazil

Pérez Gonzalez E., Brazil

Pérez-Maqueda, L., Spain

Petkova-Stoyanova, V., Bulgaria

Petkovic, M., Russian Federation

Petrič, M., Slovenia

Pielichowski, K., Poland

Plešingerová, B., Slovakia

Pop, N., Romania

Popescu, C., Germany

Poznański, J., Poland

Pratap, A., India

Prime, R. B., USA

Pruchnik, H., Poland

Rangel-Porras, G., Mexico

Reben, M., Poland

Regdon Jr., G., Hungary

Relkin, P., France

Ribeiro, C. A., Brazil

Riposan, I., Romania

Rogulska, M., Poland

Rokicki, G., Poland

Romualdez, J. L., Canada

Ronkay, F., Hungary

Rybiński, P., Poland

Rycerz, L., Poland

Rzączyńska, Z. M., Poland

Sachajdak, A., Poland

Sakizci, M., Turkey

Salunke, S. A., India

Sasca, V. Z., Romania

Sava, I., Romania

Schiraldi, A., Italy

Schnitzler, E., Brazil

Sedlářová, I., Czech Republic

Setua, D. K., India

Sieroń-Stoltny, K., Poland

Šimon, P., Slovakia

Sitarz, M. T., Poland

Skwarek, E., Poland

Sławiński, G., Poland

Slovak, V., Czech Republic

Smolik, J., Poland

Sobiecki, J. R., Poland

Soliman, M. H., Egypt

Sousa Epaminondas, P., Brazil

Spassov, T., Bulgaria

Staszczuk, P., Poland

Sternik, D., Poland

Stoia, M. E., Romania

Studzinski, M., Poland

Šulcová, P., Czech Republic

Suzuki T., Japan 
Svidró, J. T., Sweden

Svoboda, R., Czech Republic

Świątkowski, A., Poland

Szczygieł, I., Poland

Szépvölgyi, J., Hungary

Szumera, M., Poland

Tabero, P., Poland

Terekhova, I. V., Russian Federation

Thakur, V. K., USA

Tiverios, V., Greece

Toledo Bonemer de Salvi, D., Brazil

Tomaszewicz, E., Poland

Tomaszewska-Gras, J., Poland

Tudor Popa, V., Romania

Vecchio Ciprioti, S., Italy

Vinu, R., India

Vladimirovna Boldyreva, E., Russian Federation

Wacławska, I., Poland

Wang, F., China

Wang, N., China

Wang, X., China

Wei, P., China

Wesołowski, M., Poland
Wiecińska, P., Poland

Wilińska, I., Poland

Wirawan, R., Indonesia

Wojakowska, A., Poland

Worzakowska, M., Poland

Wu, K., China

Wu, S.-H., Taiwan

Xiao, Z., China

$\mathrm{Xu}, \mathrm{S} .$, China

Xu, Y., China

Yesilkaynak, T., Turkey

You, M.-L., Taiwan

Zdravkovic, J., Russian Federation

Zivanovic, V., Russian Federation

Živkovič, Ž., Serbia

Open Access This article is distributed under the terms of the Creative Commons Attribution 4.0 International License (http://crea tivecommons.org/licenses/by/4.0/), which permits unrestricted use, distribution, and reproduction in any medium, provided you give appropriate credit to the original author(s) and the source, provide a link to the Creative Commons license, and indicate if changes were made. 